\title{
RESIDU DE GROTHENDIECK ET FORME DE CHOW
}

\author{
M. ElKADI
}

\begin{abstract}
We show an explicit relation between the Chow form and the Grothendieck residue; and we clarify the role that the residue can play in the intersection theory besides its role in the division problem.
\end{abstract}

\section{Introduction}

Soit $I$ un idéal homogène de $\mathbf{Z}\left[Z_{0}, \ldots, Z_{n}\right]$. Pour tout entier $r, 1 \leq r \leq$ $n$, on introduit des formes linéaires en $Z=\left(Z_{0}, \ldots, Z_{n}\right)$ à coefficients indéterminés

$$
L_{i}(Z)=U_{i, 0} Z_{0}+\ldots+U_{i, n} Z_{n}, \quad 1 \leq i \leq r .
$$

On note $\mathbf{Z}[U]$ l'anneau des polynômes à coefficients entiers en les variables

$$
U=\left(U_{i, j} ; 1 \leq i \leq r, 0 \leq j \leq n\right)
$$

et on définit l'idéal $I(r)$ de $\mathbf{Z}[U]$ par

$$
I(r)=\left\{P \in \mathbf{Z}[U]: \exists N \in \mathbf{N}, Z_{i}^{N} P \in\left(L_{1}, \ldots, L_{r}, I\right), 0 \leq i \leq n\right\}
$$

où $\left(L_{1}, \ldots, L_{r}, I\right)$ désigne l'idéal de $\mathbf{Z}[U][Z]$ engendré par les formes linéaires $L_{1}, \ldots, L_{r}$ et les éléments de $I$. Lorsque $I$ est équidimensionnel de rang $n+1-r, I(r)$ est un idéal principal non réduit à $\{0\}$ ([N1]). Un générateur de $I(r)$ est appelé forme de Chow entière (integral Chow form) de l'idéal $I$; c'est un polynôme homogène par rapport à chaque bloc de variables $U_{i}=\left(U_{i, 0}, \ldots, U_{i, n}\right), 1 \leq i \leq r$. On renvoie à $([\mathbf{C}]$, [HP]) pour les propriétés des formes de Chow.

Dans la pratique, il est difficile, en général, de calculer la forme de Chow $\Phi$ d'un idéal donné $I=\left(P_{1}, \ldots, P_{m}\right)$. Néanmoins, les estimations 
suivantes de son degré et de la taille de ses coefficients sont contenues dans les travaux ([B2], [N1], [N2])

$$
\operatorname{deg}_{U_{i}} \Phi \leq D_{1} \ldots D_{m}, 1 \leq i \leq r,
$$

$$
h(\Phi) \leq \sum_{j=1}^{m} h_{j} D_{1} \ldots D_{j-1} D_{j+1} \ldots D_{m}+3 n^{2} m D_{1} \ldots D_{m}
$$

où $D_{j}=\operatorname{deg} P_{j}$ et $h_{j}=h\left(P_{j}\right)=\log \max \left|\operatorname{coeff}\left(P_{j}\right)\right|, 1 \leq j \leq m$.

Brownawell a repris les travaux de Nesterenko ([N1], [N2]) sur les formes de Chow pour rendre effectives les inégalités de type Lojasiewicz globales dans le cas algébrique ([B1], [B2]); et c'est précisément en exploitant ces inégalités qu'il a donné une version effective "économique" du Nullstellensatz ([B1]).

$\mathrm{Si}$

$$
I=\mathcal{D}_{1} \cap \ldots \cap \mathcal{D}_{s}
$$

est la décomposition primaire de l'idéal homogène et équidimensionnel $I$ et $\mathcal{P}_{1}, \ldots, \mathcal{P}_{s}$ ses composantes premières associées, nous désignons par $Z\left(\mathcal{P}_{i}\right)$ la variété des zéros de l'idéal premier $\mathcal{P}_{i}$ et $e\left(\mathcal{D}_{i}\right)$ l'exposant de l'idéal primaire $\mathcal{D}_{i}$ (i.e. le plus petit entier positif $m_{i}$ tel que $\mathcal{P}_{i}^{m_{i}} \subset \mathcal{D}_{i}$ ). Dans $([\mathbf{P}])$, Philippon a utilisé la forme de Chow de l'idéal $I$ pour mesurer la complexité arithmétique du cycle $c=\sum_{i=1}^{s} e\left(\mathcal{D}_{i}\right) Z\left(\mathcal{P}_{i}\right)$.

Le but de cet article est de mettre en lumière le rôle que le résidu de Grothendieck peut jouer dans la théorie de l'intersection, en explicitant son lien avec la forme de Chow, puis d'éclaircir son rôle dans le processus de division; ainsi le résidu de Grothendieck peut être utilisé comme outil pour mesurer la complexité arithmétique.

Pour finir cette introduction, nous rappelons la définition et quelques propriétés du résidu de Grothendieck ([BGVY], $[\mathbf{G H}],[\mathbf{T}])$ qui sera l'outil de base dans ce que nous développerons par la suite.

Définition 1.3. Soient $P_{1}, \ldots, P_{n} \in \mathbf{C}\left[Z_{1}, \ldots, Z_{n}\right]$ définissant une variété algébrique $V$ discrète (donc finie) et $Q$ un autre polynôme. On appelle résidu de Grothendieck local en un point $a \in V$, associé à l'application $P=\left(P_{1}, \ldots, P_{n}\right)$, de la forme différentielle holomorphe $Q(\zeta) d \zeta\left(\zeta=\left(\zeta_{1}, \ldots, \zeta_{n}\right)\right)$ le nombre

$$
\langle\bar{\partial}(1 / P), Q(\zeta) d \zeta\rangle_{a}=\left(\frac{1}{2 \pi i}\right)^{n} \int_{\Gamma_{\epsilon}} \frac{Q(\zeta)}{P_{1}(\zeta) \ldots P_{n}(\zeta)} d \zeta
$$


où $\Gamma_{\epsilon}$ est le $n$-cycle $\left\{\zeta \in U_{a}:\left|P_{i}(\zeta)\right|=\epsilon, 1 \leq i \leq n\right\}\left(U_{a}\right.$ étant un voisinage de $a$ ), qui est régulier pour $\epsilon>0$ suffisament petit et à l'extérieur d'un ensemble de mesure nulle défini par le théorème de Sard. L'orientation de $\Gamma_{\epsilon}$ est déterminée par la condition $\mathrm{d}\left(\arg P_{1}\right) \wedge \ldots \wedge$ $\mathrm{d}\left(\arg P_{n}\right) \geq 0$. Il est bien connu que la quantité à droite de (1.4) ne dépend pas de $\epsilon$ (grâce à la formule de Stokes).

Le résidu de Grothendieck global, associé à $P$, de la $n$-forme $Q(\zeta) d \zeta$, noté $\langle\bar{\partial}(1 / P), Q(\zeta) d \zeta\rangle$, est la somme des résidus locaux aux différents points de $V$, soit

$$
\langle\bar{\partial}(1 / P), Q(\zeta) d \zeta\rangle=\sum_{a \in V}\langle\bar{\partial}(1 / P), Q(\zeta) d \zeta\rangle_{a}
$$

Nous rappelons les deux propriétés fondamentales du résidu de Grothendieck, la loi de transformation et le théorème de dualité.

Proposition 1.5 (Loi de transformation). Soit $p=\left(p_{1}, \ldots, p_{n}\right)$ une autre application polynomiale définissant une variété discrète telle que

$$
\forall i=1, \ldots, n, \quad p_{i}=\sum_{j=1}^{n} a_{i, j} P_{j}, \quad a_{i, j} \in \mathbf{C}\left[Z_{1}, \ldots, Z_{n}\right]
$$

alors,

$$
\langle\bar{\partial}(1 / P), Q(\zeta) d \zeta\rangle=\left\langle\bar{\partial}(1 / p), Q(\zeta) \operatorname{det}\left(a_{i, j}(\zeta)\right) d \zeta\right\rangle
$$

Via la théorie de l'élimination, l'idéal engendré par $P_{1}, \ldots, P_{n}$ contient des polynômes $p_{1}, \ldots, p_{n}$, où $p_{i}$ dépend seulement de la variable $z_{i}$ (car la variété algébrique définie par $P_{1}, \ldots, P_{n}$ est discrète); en utilisant la formule (1.6), le calcul du résidu multidimensionnel se ramène au calcul usuel d'une seule variable.

Proposition 1.7 (Théorème de dualité). Soit $R$ un polynôme à $n$ variables à coefficients complexes; alors $R$ appartient à l'idéal engendré par $P_{1}, \ldots, P_{n}$ si, et seulement si, $\langle\bar{\partial}(1 / P), Q(\zeta) R(\zeta) d \zeta\rangle=0$ pour tout élément $Q$ de $\mathbf{C}\left[Z_{1}, \ldots, Z_{n}\right]$.

Le résidu de Grothendieck permet donc de tester l'appartenance d'un polynôme à un idéal.

Je tiens à remercier Alain Yger, Carlos Berenstein et Paul Pedersen pour l'aide qu'ils m'ont apporté. 


\section{Lien entre le résidu de Grothendieck et la forme de Chow}

Proposition 2.1. (voir aussi la Proposition 5.15 de [BGVY]). Soit I un idéal homogène équidimensionnel de $\mathbf{Z}\left[Z_{0}, \ldots, Z_{n}\right]$ engendré par des polynômes $P_{1}, \ldots, P_{m}$ définissant une suite régulière et $P_{m+1}, \ldots, P_{n+1}$ des formes linéaires génériques de la forme (1.1). Posons $P=\left(P_{1}, \ldots, P_{n+1}\right), \gamma=\operatorname{deg} P_{1}+\ldots+\operatorname{deg} P_{m}-m+1$ et

$$
\langle k, D\rangle=k_{1} \operatorname{deg} P_{1}+\ldots+k_{m} \operatorname{deg} P_{m}+k_{m+1}+\ldots+k_{n+1}
$$

pour tout multi-indice $k=\left(k_{1}, \ldots, k_{n+1}\right) \in \mathbf{N}^{n+1}$; alors un dénominateur commun aux fractions rationnelles en $U=\left(U_{i, j} ; m+1 \leq i \leq n+1\right.$, $0 \leq j \leq n)$

$$
\left\langle\bar{\partial}\left(1 / P^{k+1}\right), \zeta^{l} d \zeta\right\rangle, \quad 1 \leq\langle k, D\rangle \leq \gamma, \quad \gamma \leq l \leq 2 \gamma-1,
$$

où $\underline{1}=(1, \ldots, 1)$, est une puissance de la forme de Chow $\Phi$ de l'idéal $I$; de fait $\Phi^{\gamma+n+1}$ est un dénominateur des résidus (2.2).

Preuve: Pour un choix générique des formes linéaires $P_{m+1}, \ldots, P_{n+1}$, on a

$$
\{0\}=\left\{Z \in \mathbf{C}^{n+1}: P_{1}(Z)=\ldots=P_{n+1}(Z)=0\right\} .
$$

Il s'ensuit que l'idéal $\left(Z_{0}, \ldots, Z_{n}\right)^{\gamma}$ est contenu dans $\left(P_{1}, \ldots, P_{n+1}\right)$. En effet, soient $h$ un monôme de degré $\gamma$ et $g$ un monôme quelconque

$$
\langle\bar{\partial}(1 / P), g(\zeta) h(\zeta) d \zeta\rangle=\lim _{s \rightarrow \infty}\left(\frac{1}{2 \pi i}\right)^{n+1} \int_{\Gamma_{s}} \frac{h(\zeta) g(\zeta)}{P_{1}(\zeta) \ldots P_{n+1}(\zeta)} d \zeta
$$

avec $\Gamma_{s}=\left\{\zeta \in \mathbf{C}^{n+1}:\left|P_{i}(\zeta)\right| \sim \frac{1}{s^{\operatorname{deg} P_{i}}}, 1 \leq i \leq n+1\right\}$. En faisant le changement de variable homothétique $u=s \zeta$, on voit que la quantité (2.3) est nulle. D'après le théorème de dualité (Proposition 1.7) $h$ est dans l'idéal engendré par $P_{1}, \ldots, P_{n+1}$.

L'ensemble $B(R)=B\left(R_{1}, \ldots, R_{n+1}\right)=\left\{\zeta \in \mathbf{C}^{n+1}:\left|P_{i}(\zeta)\right|<R_{i}\right.$, $1 \leq i \leq n+1\}$ est un polyèdre de Weil pour presque tous les choix des nombres réels strictement positifs $R_{1}, \ldots, R_{n+1}$ grands. A l'intérieur de celui-ci, nous écrirons pour tout $j \in\{0, \ldots, n\}$, grâce à la formule de Weil ([AY], [BGVY])

$$
Z_{j}^{\gamma}=\left(\frac{1}{2 \pi i}\right)^{n+1} \int_{\Gamma(R)} \frac{\zeta_{j}^{\gamma} H(\zeta, Z)}{\prod_{i=1}^{n+1}\left(P_{i}(\zeta)-P_{i}(Z)\right)} d \zeta
$$


où $\Gamma(R)=\left\{\zeta \in \mathbf{C}^{n+1}:\left|P_{i}(\zeta)\right|=R_{i}, 1 \leq i \leq n+1\right\}$ est la frontière de Shilov de $B(R)$ et $H(\zeta, Z)$ est le déterminant d'une matrice de polynômes en $2 n+2$ variables

$$
\left(P_{i, j}(\zeta, Z) ; 1 \leq i \leq n+1,0 \leq j \leq n\right)
$$

formant un système de diviseurs de Hefer associé à l'application $P=$ $\left(P_{1}, \ldots, P_{n+1}\right)$, soit

$\forall i=1, \ldots, n+1, \forall \zeta, Z \in \mathbf{C}^{n+1}, P_{i}(\zeta)-P_{i}(Z)=\sum_{j=1}^{n} P_{i, j}(\zeta, Z)\left(\zeta_{j}-Z_{j}\right)$,

par exemple, nous pouvons prendre

$$
P_{i, j}(\zeta, Z)=\frac{P_{i}\left(Z_{1}, \ldots, Z_{j-1}, \zeta_{j}, \ldots, \zeta_{n}\right)-P_{i}\left(Z_{1}, \ldots, Z_{j}, \zeta_{j+1}, \ldots, \zeta_{n}\right)}{\zeta_{j}-Z_{j}} .
$$

En développant en série géométrique le noyau intégral de (2.4), nous obtenons

(2.5) $Z_{j}^{\gamma}=\left(\frac{1}{2 \pi i}\right)^{n+1} \sum_{k \in \mathrm{N}^{n+1}}\left(\int_{\Gamma(R)} \frac{\zeta_{j}^{\gamma} H(\zeta, Z)}{\prod_{i=1}^{n+1} P_{i}^{k_{i}+1}(\zeta)} d \zeta\right) \prod_{i=1}^{n+1} P_{i}^{k_{i}}(Z)$.

Le membre de droite de (2.5) peut s'exprimer en terme du résidu de Grothendieck,

$$
Z_{j}^{\gamma}=\sum_{k \in \mathrm{N}^{n+1}}\left\langle\bar{\partial}\left(1 / P^{k+1}\right), \zeta_{j}^{\gamma} H(\zeta, Z) d \zeta\right\rangle \prod_{i=1}^{n+1} P_{i}^{k_{i}}(Z) .
$$

D'après les identités de Jacobi ([BY3]) les termes de la série figurant dans la formule (2.6) s'annulent dès que $\langle k, D\rangle>\gamma$. Donc l'identité (2.6) devient

(2.7) $\quad Z_{j}^{\gamma}=\sum_{\left\{k \in \mathrm{N}^{n+1}, 1 \leq\langle k, D\rangle \leq \gamma\right\}}\left\langle\bar{\partial}\left(1 / P^{k+\underline{1}}\right), \zeta_{j}^{\gamma} H(\zeta, Z) d \zeta\right\rangle \prod_{i=1}^{n+1} P_{i}^{k_{i}}(Z)$

car le résidu relatif à l'application polynomiale $P$ de composantes $P_{1}, \ldots, P_{n+1}$, annule l'idéal engendré par ces mêmes composantes (Théorème de dualité).

Les résidus (2.2) qui interviennent dans la formule (2.7) sont des fractions rationnelles en $U=\left(U_{i, j} ; m+1 \leq i \leq n+1,0 \leq j \leq n\right)$ à coefficients entiers. Un dénominateur commun quelconque de ces fractions 
est un multiple de la forme de Chow $\Phi$ de l'idéal $I=\left(P_{1}, \ldots, P_{m}\right)$. D'autre part, d'après la définition de $\Phi$, il existe un entier positif $N$ tel que

$$
\forall j=0, \ldots, n, \quad \Phi(U) \zeta_{j}^{N} \in\left(P_{1}, . ., P_{n+1}\right) .
$$

Il en découle alors que l'on peut trouver des polynômes $Q_{j, 1}(U, Z), \ldots, Q_{j, n+1}(U, Z)$ à coefficients entiers en les variables $(U, \zeta)$ satisfaisant

$$
\Phi(U) \zeta_{j}^{N}=Q_{j, 1}(U, Z) P_{1}(Z)+\ldots+Q_{j, n+1}(U, Z) P_{n+1}(Z), 0 \leq j \leq n .
$$

En utilisant la loi de transformation du résidu ([Ky]), les expressions (2.2) sont égales à

$$
\begin{aligned}
\frac{1}{\Phi(U)^{|k|+n+1}} \sum_{0 \leq r_{1}, \ldots, r_{|k|} \leq n} & \frac{c_{r_{1}, \ldots, r_{|k|}}}{(k+\underline{1}) !}\left(\frac{1}{2 \pi i}\right)^{n+1} \\
& \times \int_{C_{R}} \frac{\zeta^{l} \operatorname{det}\left(Q_{i, j}(U, \zeta)\right) Q_{r_{1}, \ldots, r_{|k|}}(U, \zeta)}{\zeta_{0}^{N\left(i_{0}+1\right)} \ldots \zeta_{n}^{N\left(i_{n}+1\right)}} d \zeta
\end{aligned}
$$

où $k=\left(k_{1}, \ldots, k_{n+1}\right) \in \mathbf{N}^{n+1},|k|=k_{1}+\ldots+k_{n+1}, k !=k_{1} ! \ldots k_{n+1} !$, $c_{r_{1}, \ldots, r_{|k|}}=i_{0} ! \ldots i_{n}$ ! avec $i_{j}$ est le nombre de $j$ dans la liste $\left(r_{1}, \ldots, r_{|k|}\right)$, $C_{R}=\left\{\zeta \in \mathbf{C}^{n+1}:\left|\zeta_{i}\right|=R, 0 \leq i \leq n\right\}$ et

$$
Q_{r_{1}, \ldots, r_{|k|}}(U, \zeta)=\prod_{\left\{1 \leq v \leq n+1,1 \leq i \leq k_{v}\right\}} Q_{r_{k_{1}+\ldots+k_{v-1}+i}, v}(U, \zeta) .
$$

Donc une puissance de la forme $\Phi$, en l'occurrence $\Phi(U)^{\gamma+n+1}$, est un dénominateur des fractions rationnelles (2.2).

\section{Rôle du résidu de Grothendieck dans le processus de division}

Etant donnés un sous-corps $\mathbf{K}$ de $\mathbf{C}$, un idéal $I$ de $\mathbf{K}\left[Z_{1}, \ldots, Z_{n}\right]$ engendré par des polynômes $P_{1}, \ldots, P_{m}$ et un polynôme $Q$ appartenant à $I$; l'un des problèmes fondamentaux de la géométrie algébrique effecive est la donnée d'une borne pour les degrés des candidats $Q_{1}, \ldots, Q_{m}$ vérifiant

$$
Q=Q_{1} P_{1}+\ldots+Q_{m} P_{m}, Q_{i} \in \mathbf{K}\left[Z_{1}, \ldots, Z_{n}\right]
$$

Il est bien connu, qu'en général, la complexité de ce problème est doublement exponentielle en le nombre de variables $n$ ([MM]). Néanmoins, 
des bornes simplement exponentielles ont été trouvé dans certains cas particuliers ([A] $,[\mathbf{B} \mathbf{1}],[\mathbf{B Y} \mathbf{1}],[\mathbf{C G H}],[\mathbf{D F G S}],[\mathbf{F G}],[\mathbf{K}],[\mathbf{S}])$.

Si en plus $\mathbf{K}$ est muni d'une structure arithmétique, se pose la question d'estimer la taille des coefficients des polynômes $Q_{1}, \ldots, Q_{m}$. Berenstein et Yger ont été les premiers à répondre de façon satisfaisante à cette question dans le cas classique de l'identité de Bezout ([BY2]); en effet, ils ont donné des bornes simplement exponentielles, lorsque $Q=1$, pour les deux aspects géométrique et arithmétique du problème. Tout récemment, le cas de suites régulières de $\mathbf{Z}\left[z_{1}, \ldots, z_{n}\right]$ a été résolu ([E], $[\mathbf{K P}])$. Le but de cette section est de donner de "bonnes" estimations pour le degré et la taille des coefficients des quotients lorsque l'on a affaire à une famille de $m$ polynômes homogènes à coefficients entiers définissant une suite régulière dans $\mathbf{C}\left[z_{0}, \ldots, z_{n}\right]$, et par conséquent une sous variété de codimension $m$ dans $\mathbf{P}^{n}(\mathbf{C})$.

Soient $P_{1}, \ldots, P_{m}$ des polynômes homogènes de $\mathbf{Z}\left[Z_{0}, \ldots, Z_{n}\right]$ et $Q$ un polynôme à coefficients entiers appartenant à l'idéal de $\mathbf{Q}\left[Z_{0}, \ldots, Z_{n}\right]$ engendré par $P_{1}, \ldots, P_{m}$. Supposons que l'idéal $I=\left(P_{1}, \ldots, P_{m}\right)$ définisse une intersection complète dans $\mathbf{P}^{n}(\mathbf{C})$ et notons $\Phi$ la forme de Chow de $I$. Nous voulons diviser explicitement $Q$ dans $\left(P_{1}, \ldots, P_{m}\right)$. Les fractions rationnelles du type (2.2) sont appelées à jouer un rôle.

Proposition 3.1. Sous ces hypothèses, il existe des polynômes

$$
A_{1}(U, Z), \ldots, A_{m}(U, Z) \in \mathbf{Z}[U, Z]
$$

de degrés par rapport à $Z$ au plus $\operatorname{deg} Q+\operatorname{deg} P_{1}+\ldots+\operatorname{deg} P_{m}-m$, par rapport à chaque bloc de variables $U_{i}, m+1 \leq i \leq n+1$, au plus $(\operatorname{deg} Q+n+1) D_{1} \cdots D_{m}$, tels que

$$
\Phi^{\operatorname{deg} Q+n+1}(U) Q(Z)=A_{1}(U, Z) P_{1}(Z)+\ldots+A_{m}(U, Z) P_{m}(Z) .
$$

Preuve: En effet, soient $P_{m+1}, \ldots, P_{n+1}$ des formes linéaires comme dans la Proposition 2.1. De nouveau grâce à la formule de Weil, nous écrịvons dans le domaine de Weil $B(R)$

$$
Q(Z)=\left(\frac{1}{2 \pi i}\right)^{n+1} \int_{\Gamma(R)} \frac{Q(\zeta) H(\zeta, Z)}{\prod_{i=1}^{n+1}\left(P_{i}(\zeta)-P_{i}(Z)\right)} d \zeta
$$

D'autre part, comme le polynôme $Q$ appartient à l'idéal engendré par $P_{1}, \ldots, P_{m}$, nous avons (en utilisant le théorème de dualité)

$$
0=\left(\frac{1}{2 \pi i}\right)^{n+1} \int_{\Gamma(R)} \frac{Q(\zeta) H(\zeta, Z)}{\prod_{i=1}^{n+1} P_{i}(\zeta)} d \zeta
$$


Suivant ([BT, Proposition 1.3]), nous soustrayons (3.3) de (3.2), et nous trouvons

$$
Q(Z)=\sum_{s=1}^{m} \sum_{\substack{s \subset\{1, \ldots, m\} \\ \text { card } S=s}}\left(\prod_{i \in S} P_{i}(Z)\right) A_{S}(Z)
$$

avec,

$$
\begin{aligned}
& A_{S}(Z)=\left(\frac{1}{2 \pi i}\right)^{n+1} \\
& \times \int_{\Gamma(R)} \frac{Q(\zeta) H(\zeta, Z)}{\prod_{i=1}^{m} P_{i}(\zeta) \prod_{i=m+1}^{n+1}\left(P_{i}(\zeta)-P_{i}(Z)\right) \prod_{i \in S}\left(P_{i}(\zeta)-P_{i}(Z)\right)} d \zeta .
\end{aligned}
$$

En développant en série géométrique le noyau intégral de $A_{S}$, nous obtenons

$$
\begin{aligned}
& \sum_{\substack{k \in \mathbb{N}^{s} \\
l \in \mathbb{N}^{n+1-m}}}\left(\left(\frac{1}{2 \pi i}\right)^{n+1}\right. \\
\times & \left.\int_{\Gamma(R)} \frac{Q(\zeta) H(\zeta, Z)}{\prod_{i \notin S} P_{i}(\zeta) \prod_{i \in S} P_{i}^{k_{i}+2}(\zeta) \prod_{i=m+1}^{n+1} P_{i}^{l_{i}+1}(\zeta)} d \zeta\right) F_{S, k, l}(Z)
\end{aligned}
$$

avec,

$$
F_{S, k, l}(Z)=\left(\prod_{i \in S} P_{i}^{k_{i}}(Z)\right)\left(\prod_{i=m+1}^{n+1} P_{i}^{l_{i}}(Z)\right) .
$$

En exprimant les termes entre parenthèses dans (3.4) en termes de résidus, nous avons

$$
A_{S}(Z)=\sum_{\substack{k \in \mathbb{N}^{s} \\ l \in \mathbb{N}^{n+1-m}}}\left\langle\bar{\partial}\left(1 / P^{\epsilon(S, k, l)}\right), Q(\zeta) H(\zeta, Z) d \zeta\right\rangle F_{S, k, l}(Z)
$$

où $\epsilon_{i}(S, k, l)=1, i \notin S ; \epsilon_{i}(S, k, l)=k_{i}+2, i \in S ; \epsilon_{i}(S, k, l)=l_{i}+$ $1, m+1 \leq i \leq n+1$.

D'après les identités de Jacobi ([BY3]) les termes de la série (3.5) s'annulent dès que

$$
\sum_{i \in S}\left(k_{i}+1\right) \operatorname{deg} P_{i}+\sum_{i=m+1}^{n+1} l_{i}>\operatorname{deg} Q .
$$


Il en résulte que

$$
\begin{array}{r}
Q(Z)=\sum_{s=1}^{m} \sum_{\substack{\{\subset\{1, \ldots, m\} \\
\operatorname{card}(S)=s}} \sum_{k, l}\left(\prod_{i \in S} P_{i}(Z)\right) \\
\times\left\langle\bar{\partial}\left(1 / P^{\epsilon(S, k, l)}\right), Q(\zeta) H(\zeta, Z) d \zeta\right\rangle F_{S, k, l}(Z)
\end{array}
$$

où la somme sur les multi-indices $k \in \mathbf{N}^{s}, l \in \mathbf{N}^{n+1-m}$ se réduit à

$$
\sum_{i \in S}\left(k_{i}+1\right) \operatorname{deg} P_{i}+\sum_{i=m+1}^{n+1} l_{i} \leq \operatorname{deg} Q .
$$

Les quantités

$$
\left\langle\bar{\partial}\left(1 / P^{\epsilon(S, k, l)}\right), \zeta^{d} d \zeta\right\rangle, \quad|d| \leq \operatorname{deg} Q+\operatorname{deg} P_{1}+\ldots+\operatorname{deg} P_{m}-m
$$

qui interviennent dans l'identité (3.6) sont des fractions rationnelles en $U$ à coefficients entiers. Nous venons de voir, dans la section 2 , que $\Phi^{\operatorname{deg} Q+n+1}$ est un dénominateur commun à ces fractions rationnelles. Il en découle alors que

$$
\begin{aligned}
& \Phi^{\operatorname{deg} Q+n+1}(U) Q(Z) \\
& \quad=\tilde{A}_{1}(U, Z) P_{1}(Z)+\ldots+\tilde{A}_{m}(U, Z) P_{m}(Z), \tilde{A}_{i} \in \mathbf{Z}[U, Z],
\end{aligned}
$$

avec

$$
\operatorname{deg}_{Z} \tilde{A}_{i} \leq \operatorname{deg} Q+\operatorname{deg} P_{1}+\ldots+\operatorname{deg} P_{m}-m .
$$

On obtient les polynômes $A_{i}$ en ne conservant dans le membre de droite que les parties homogènes en $U$ de degré le degré de $\Phi^{\operatorname{deg} Q+n+1}$, soit $(\operatorname{deg} Q+n+1) D_{1} \cdots D_{m}$.

Ceci montre que le numérateur du résidu joue un rôle dans le processus de division tandis que son dénominateur joue un rôle dans la théorie de l'intersection.

Proposition 3.8. Soient $P_{1}, \ldots, P_{m}$ des polynômes homogénes de $\mathbf{Z}\left[Z_{0}, \ldots, Z_{n}\right]$ de degrés respectifs au plus $D_{1}, \ldots, D_{m}$ et de hauteurs logarithmiques au plus $h$. Supposons que $P_{1}, \ldots, P_{m}$ forment une intersection complète de $\mathbf{P}^{n}(\mathbf{C})$. Posons $\Delta=D_{1} \cdots D_{m}$ et $D=\max \left(D_{1}, \ldots, D_{m}\right)$; alors pour tout polynôme $Q$ à coefficients entiers appartenant à l'idéal de $\mathbf{Q}\left[Z_{0}, \ldots, Z_{n}\right]$ engendré par $P_{1}, \ldots, P_{m}$, il existe des polynômes $A_{1}, \ldots, A_{m}$ appartenant à $\mathbf{Z}\left[Z_{0}, \ldots, Z_{n}\right]$ et un entier $\delta$ strictement positif tels que

$$
\delta Q=A_{1} P_{1}+\ldots+A_{m} P_{m}
$$


avec les estimations suivantes

$$
\begin{gathered}
\operatorname{deg} A_{i} \leq \operatorname{deg} Q+D_{1}+\ldots+D_{m}-m \\
\log \delta \leq \kappa(n, m)(\operatorname{deg} Q+n+1) \Delta(h+\log D+1) \\
h\left(A_{i}\right) \leq \kappa(n)\left((\operatorname{deg} Q+1)\left(D \Delta+h \Delta+\log \left(D_{Q}+1\right)+h_{Q}\right)\right.
\end{gathered}
$$

avec $\kappa(n, m)=n^{3}+n^{2} m+3 n^{2}+n m^{2}$ et $\kappa(n)$ étant une constante effectivement calculable ne dépendant que du nombre de variables $n$.

Preuve: Comme le degré de la forme de Chow $\Phi$ de $\left(P_{1}, \ldots, P_{m}\right)$ par rapport à chaque bloc de variables $U_{i}=\left(U_{i, 0}, \ldots, U_{i, n}\right), m+1 \leq i \leq$ $n+1$, est au plus $\Delta$, il existe, d'après un lemme de zéro immédiat, un choix de $u=\left(u_{m+1}, \ldots, u_{n+1}\right) \in\left(\mathbf{Z}^{n+1}\right)^{n+1-m}$ tel que $\left|u_{i, j}\right| \leq \Delta$ et $\Phi(u) \neq 0$. Nous faisons ce choix et posons

$$
\delta=|\Phi(u)|^{\operatorname{deg} Q+n+1}
$$

nous avons l'estimation suivante pour $\log \delta$ (notons $c=n+1-m$ ),

$$
(\operatorname{deg} Q+n+1)(h(\Phi)+((n-1) c-1) \log (1+c \Delta)+c \Delta \log \Delta) .
$$

En utilisant l'estimation (1.2), nous trouvons la borne désirée pour le dénominateur $\delta$.

Il nous reste à estimer la taille des coefficients de $A_{1}, \ldots, A_{m} \in$ $\mathbf{Z}\left[Z_{0}, \ldots, Z_{n}\right], A_{i}(Z)=A_{i}(u, Z)$; pour cela nous allons chercher une borne pour les nombres rationnels (3.7) qui figurent dans l'expression de $A_{1}, \ldots, A_{m}$. Les résidus (3.7) peuvent s'écrire sous la forme

$$
\begin{aligned}
& \frac{(-1)^{n(n+1) / 2}(|\epsilon|-1) !}{(2 \pi i)^{n+1}(\epsilon-1) !} \\
& \quad \times \int_{\|\zeta\|=r} \frac{\bar{P}_{1}^{\epsilon_{1}-1} \ldots \bar{P}_{n+1}^{\epsilon_{n+1}-1} \sum_{i=1}^{n+1}(-1)^{i-1} \bar{P}_{i} \overline{d P}_{[i]} \wedge \zeta^{d} d \zeta}{\|P(\zeta)\|^{2|\epsilon|}}
\end{aligned}
$$

où $d P_{[i]}=d P_{1} \wedge \ldots \wedge d P_{i-1} \wedge d P_{i+1} \wedge \ldots \wedge d P_{n}, 1 \leq i \leq n$. En appliquant l'inégalité de type Lojasiewicz établie par Brownawell ([B2, Théorème A]) à $\zeta \in \mathbf{C}^{n},\|\zeta\|=r$ et $P=\left(P_{1}, \ldots, P_{n+1}\right)$ nous obtenons

(3.9) $\quad \log \|P(\zeta)\|$

$$
\geq-\Delta\left\{11(n+2)^{5} D+(n+2)^{2} \max (h, \log \Delta)\right\}-(n+2)^{2} \Delta \log \left(|\zeta|^{2} / \rho\right)
$$


où $|\zeta|=\max \left(1, \zeta_{0}, \ldots, \zeta_{n}\right)$ et $0<\rho \leq 1$. L'inégalité (3.9) fournit la borne suivante pour les nombres rationnels (3.7)

$$
\exp (\kappa(n)(\operatorname{deg} Q+1)(D \Delta+h \Delta+\log (\operatorname{deg} Q+1)))
$$

avec $\kappa(n)$ une constante effective ne dépendant que de $n$. Comme nous savons estimer la taille des coefficients des autres polynômes qui interviennent dans l'écriture de $A_{1}, \ldots, A_{m}$, nous déduisons la borne donnée dans la Proposition 3.8 pour les hauteurs logarithmiques des quotients $A_{1}, \ldots, A_{m}$.

Remarque 3.10. L'existence de bornes du type (3.8) pour les hauteurs des quotients $A_{i}, 1 \leq i \leq m$, et du dénominateur $\delta$ résulterait aisément d'un argument d'algèbre linéaire (compte tenu de l'estimation à priori concernant les degrés de ces polynômes). Ce que nous montrons avec cette proposition est qu'il est possible d'ajuster les formules de manière à ce que l'estimation du dénominateur soit en un sens optimale (le plus proche possible des estimations des coefficients de la forme de Chow).

\section{Références}

[A] F. Amoroso, Tests d'appartenance d'après un théorème de Kollár, C. R. A. S. 309 (1989), 691-694.

[AY] L. A. AǏZenberg, A. P. YuzhaKov, "Integral representations and residues in multidimensional complex analysis," American Mathematical Society, Providence, Rhode Island, 1983.

[B1] W. D. Brownawell, Bounds for the degrees in the Nullstellensatz, Ann. of Math. 126 (1987), 577-591.

[B2] W. D. BRownAwELL, Local diophantine nullstellen inequalites, J. Amer. Math. Soc. 1 (1988), 311-322.

[BGVY] C. A. Berenstein, R. Gay, A. Vidras, A. Yger, "Residue currents and Bezout identities," Progress in Math., Birkhäuser, 1993.

[BT] C. A. Berenstein, B. A. TAYLOR, Interpolation problems in $\mathbf{C}^{n}$ with applications to harmonic analysis, J. Anal. Math. 38 (1980), 188-254.

[BY1] C. A. Berenstein, A. Yger, Bounds for the degrees in the division problem, Michigan Math. J. 37 (1990), 25-43.

[BY2] C. A. Berenstein, A. YGer, Effective Bezout identities in $\mathbf{Q}\left[z_{1}, \ldots, z_{n}\right]$, Acta Math. 166 (1991), 69-120. 
[BY3] C. A. Berenstein, A. Yger, Une formule de Jacobi et ses conséquences, Ann. Sc. Ec. Norm. Sup. Paris 24 (1991), 363-377.

[C] L. Caniglia, How to compute the Chow forme of an unmixed polynomial ideal in simple exponential time, AAECC 1 (1990), $25-41$.

[CGH] L. Caniglia, M. Giusti, J. Heintz, Borne simple exponentielle pour les degrés dans les théorèmes des zéros sur un corps de caractéristique quelconque, C. R. A. S. 307 (1988), 255-258.

[DFGS] A. Dickenstein, N. Fitchas, M. Giusti, C. Sessa, The membership problem for unmixed polynomial ideals is solvable in single exponential time, Discrete Appl. Math. 33 (1991), 73-94.

[E] M. ElKadi, Bornes pour les Degrés et les Hauteurs dans le problème de division, Michigan Math. J. 40 (1993), 609-618.

[FG] N. Fitchas, A. Galligo, Nullstellensatz effectif et Conjecture de Serre (Théorème de Quillen-Suslin) pour le calcul formel, Math. Nachr. 149 (1990), 231-252.

[GH] P. Griffiths, J. HARRIS, "Principles of Algebraic Geometry," Wiley Interscience, New York, 1978.

[HP] W. V. D. Hodge, D. Pedoe, "An introduction to Algebraic Geometry," 2, Cambridge University Press, Cambridge, 1952.

[K] J. Kollár, Sharp effective Nullstellensatz, J. Amer. Math. Soc. 1 (1988), 963-975.

[KP] T. KRICK, L. M. PARDO, Une approche informatique pour l'approximation diophantienne, C. R. A. S. 318 (1994), 407-412.

[Ky] A. M. Kytmanov, A transformation formula for Grothendieck residues and some of its applications, Siberian Math. Journal (1989), 495-499.

[MM] E. MAYR, A. MEYER, The complexity of the word problem for commutative semigroups and polynomial ideals, Adv. in Math. 64 (1982), 305-329.

[N1] J. V. NESTERENKo, On algebraic independence on algebraic powers of algebraic numbers, Mat. Sbornik 123(165) (1984), 435-459; Math. USSR Sbornik 51 (1985), 429-454.

[N2] J. V. Nesterenko, Estimates for the orders of zeroes of functions of a certain class and applications in the theory of transcendental numbers, Izv. Akad. Nauk SSSR Ser. Mat. 41 (1977), 235-284; Math. USSR Izv. 11 (1977), 239-270.

[P] P. Philippon, Sur des hauteurs alternatives I, Math. Ann. 289 (1991), 255-283. 
[S] B. Shiffman, Degree bounds for the division problem in polynomial ideals, Michigan Math. J. 36 (1989), 163-171.

[T] A. K. TsiKH, "Multidimensional Residues and Their Applications," Translations of Mathematical Monographs 103, American Mathematical Society, 1992.

\author{
Université Paul Sabatier \\ U.F.R. M.I.G. (URA CNRS 1408) \\ 31062 Toulouse \\ FRANCE \\ e-mail: elkadi@ cict.fr
}

Primera versió rebuda el 9 de Febrer de 1994, darrera versió rebuda el 19 d'Abril de 1994 